\begin{tabular}{|c|c|c|}
\hline 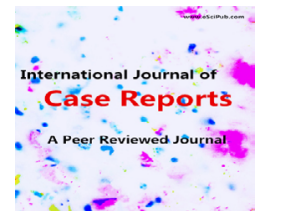 & $\begin{array}{l}\text { International Journal of Case Reports } \\
\text { (ISSN:2572-8776) }\end{array}$ & 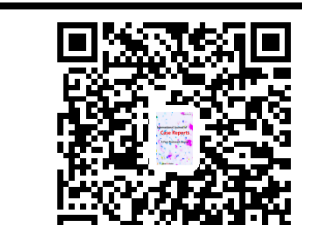 \\
\hline
\end{tabular}

\title{
A severe case of toxic epidermal necrolysis which started with AGEP-like features: Does a new clinical type of drug eruption?
}

\section{Sevil Alan ${ }^{1}$}

${ }^{1}$ Akdeniz University School of Medicine Department of Dermatology and Venereology.

\section{ABSTRACT}

Acute generalized exanthematous pustulosis (AGEP) and toxic

${ }^{*}$ Correspondence to Author:

epidermal necrolysis (TEN) are adverse cutaneous reactions. Despite the fact that these 2 cutaneous reactions differ in Sevil Alan Akdeniz University presentation, prognosis, pathologic features, and treatment, School of Medicine Department of overlap can exist between them, creating a diagnostic challenge. It has rarely been reported that AGEP overlaps or mimics TEN, while TEN which starting by multıple pustules like AGEP has not been reported. The present study reports a rare case of a 14-year-old male patient with the clinical manifestations of multiple purulent bullae caused by amoxycillin ingestion. On 3. day dermatological examination revealed flaccid bullae and epidermal splitting with positive Nikolsky's sign on right pectoral area, erosions on oral and anogenital mucosa. Histopathologic examination showed a massive inflamatory infiltration and epidermal necrosis indicated Steven Johnson syndrome. Later on added systemic symptoms with haemodynamic, respiratory imbalance and the patient was exitus. The case is discussed to reveal whether a new clinical pattern of drug eruption has been identified. We describe a patient with TEN who presented initially with clinical features of acute generalized exanthematous pustulosis (AGEP). To the best of our knowledge, one report drug eruption with purulent bulla and epidermal necrolysis has previously been documented, and therefore the present study is the second case report of its kind. Because the initial presentation of such adverse drug reactions may vary, diagnosis is difficult and dermatologists must careful the possibility of mimics among adverse cutaneous reactions. 


\section{Introduction}

Severe cutaneous adverse reactions to drugs include drug reaction with eosinophilia and sytemic symptoms (DRESS), acute generalized exanthematous pustulosis (AGEP), and epidermal necrolysis (Stevens-Johnson syndrome-toxic epidermal necrolysis (SJS-TEN)) ${ }^{[1]}$. Acute generalized exanthematous pustulosis (AGEP) is a drug eruption characterized by the great number of sterile, nonfollicular, pinhead-sized pustules arising on an edematous erythema ${ }^{[2]}$. More skin symptoms can contain facial edema and skin lesions such as "atypical" targets, purpura, blisters, or vesicles ${ }^{[3]}$. The clinical features are described by fever and spread of sterile pustules in a 2-5 days after the drug intake ${ }^{[4]}$. Mucous membranes usually are not typically involved. AGEP usually resolves once the trigger drug is no longer used. It is mostly participated by neutrophilia ${ }^{[5]}$. The drugs predicting the highest risk of AGEP are aminopenicillins, pristinamycin, antibacterial sulphonamides, hydroxychloroquine, terbinafine and diltiazem ${ }^{[4]}$. Toxic epidermal necrolysis (TEN) is a typically drug-induced, frequently fatal, mucocutaneous disease. First described by Scottish dermatologist Alan Lyell in 1956, it is also known as Lyells syndrome. TEN may result in deformity or death, with a mortality rate of $10 \%-40 \%{ }^{[3]}$. It is delayed type mucocutaneous immune reactions with the combination of extensive keratinocyte apoptosis. The clinical type of TEN depends on their degree of epidermal detachment ${ }^{[6]}$. The spectrum of TEN range from mild exanthematous skin rashes to a large amount of bullae and wide mucocutaneous peeling. The incidence of TEN and drug reactions generally is 2.7 times higher in the geriatric than in a younger population ${ }^{[3]}$. The mortality rate is up to $5 \%$ and mostly occurs in elderly people who have significant comorbidities [4]. Although AGEP and SJS/TEN are distinct entities with a different clinical findings, pathogenesis, prognosis and treatment, some studies showed that they may share some features in literature. We present a severe case of TEN, caused by amoxicillin, clinically presenting with AGEP-like features and pronounced systemic symptoms with haemodynamic, respiratory instability and resulted by death ${ }^{[6]}$.

\section{Case}

A 14 year old male was diagnosed with T-cell lymphoblastic lymphoma 4 months ago. The patient received amoxycillin tablet 8 days before his adminstration to our hospital. He presented to our hospital with fever, fatigue, weakness, severe pain throughout his skin that was aggravated by even slight touch, and a widespread non-pruritic maculopapular rash. The patient hospitalised by the pediatricians. Him dermatological examination revealed non follicular pustules were seen on eritematous patches on areas covered about half of the lower extremities (Figure 1).

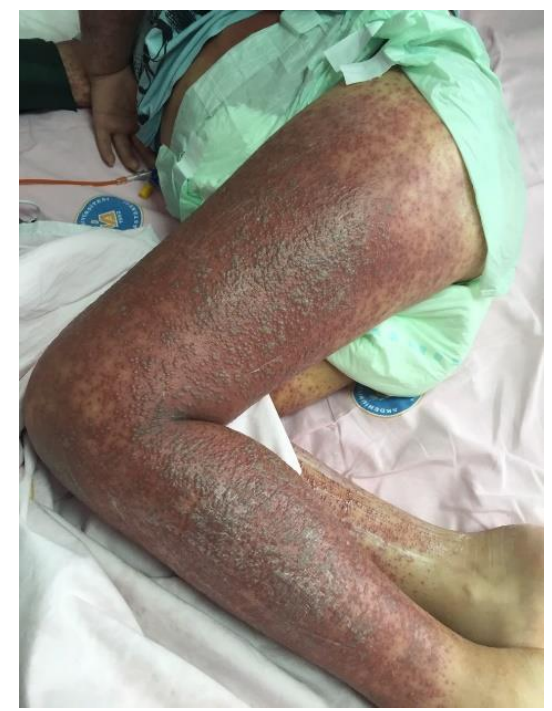

Figure legends: Figure 1. Non follicular pustules on eritematous patches on lower extremities. 
Facial oedema, eritema and oral mucosal stom- day the pustules were increasing on his occipital atitis were also determined. The clinical appear- scalp and livid eritematous plaques that covered ance was consistent with AGEP, suspicious most of the runk (Figure 2).

drugs stopped, iv fluid support beginned. On 1.

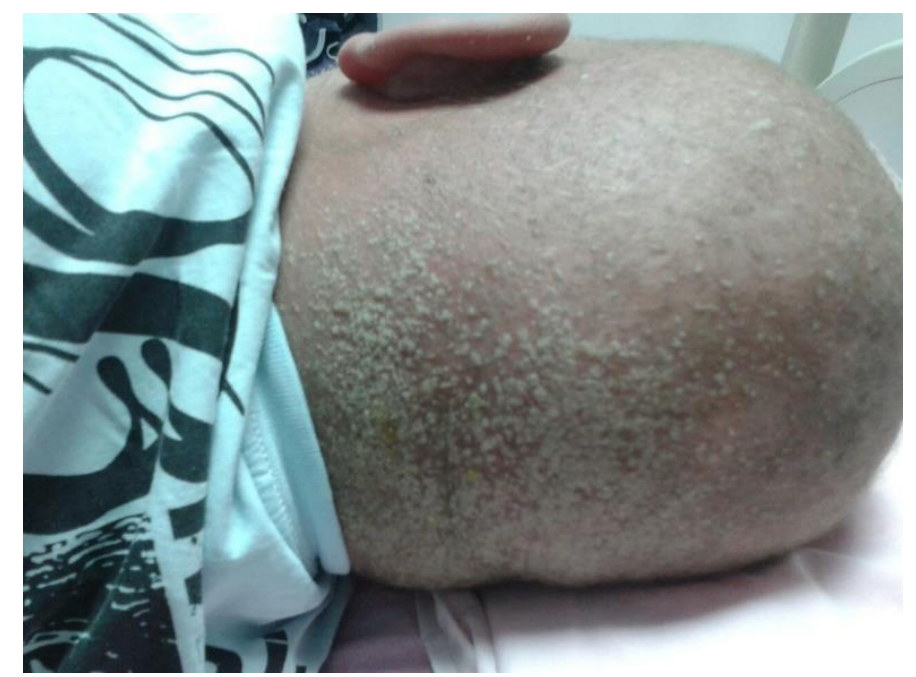

Figure 2. Multiple pustules on his occipital scalp.

We started him IV $1 \mathrm{mg} / \mathrm{kg}$ prednisolone therapy. ve Nikolsky's sign on right pectoral area, erosiOn 3. day dermatological examination revealed ons on oral and anogenital mucosa (Figure 3). flaccid bullae and epidermal splitting with positi-

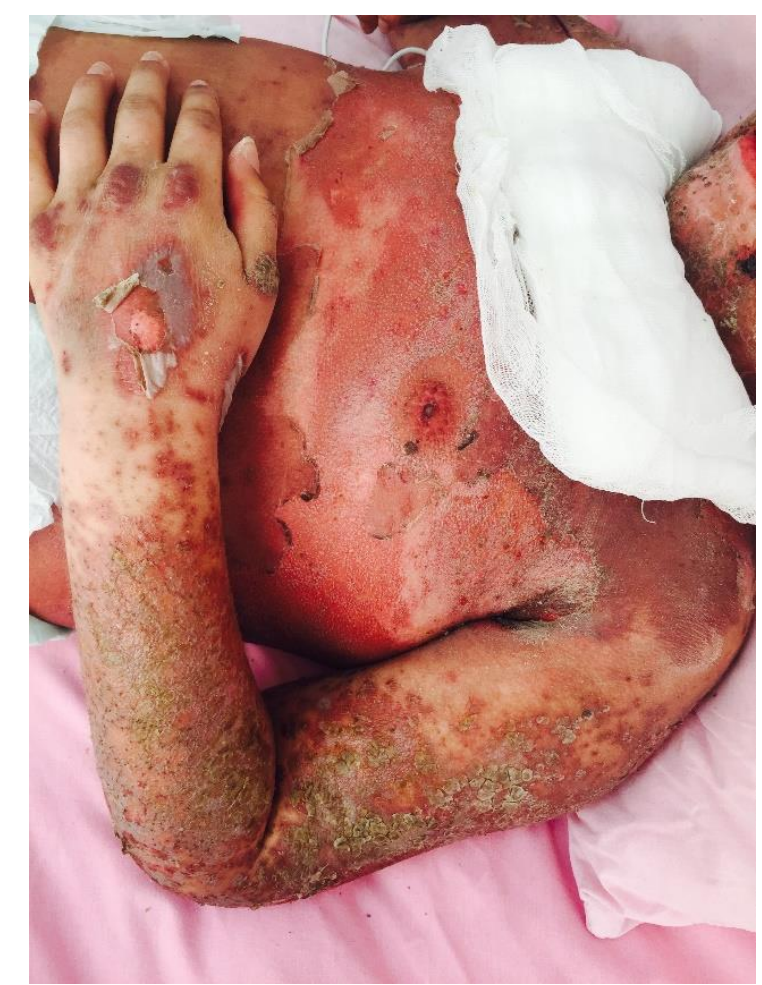

Figure 3. Flaccid bullae and epidermal splitting and erosions on him pectoral areas and upper extremities.

Ocular mucosa was normal except a few tiny hemorrhagic crusts on the eyelids. Cultures from the pustules showed no pathological microorganismas. We performed 3 biopsies: one of them was taken from right pectoral bullae with Steven Johnson syndrome as a preliminary diagnosis; one of them was taken from the left lomber pustules with AGEP as a preliminary 
diagnosis and one of them for the direct immunflorecanse examination. Histopathologic examination of first and second speciemen showed a massive inflamatory infiltration and epidermal necrosis indicated Steven Johnson syndrome (Figure 4).

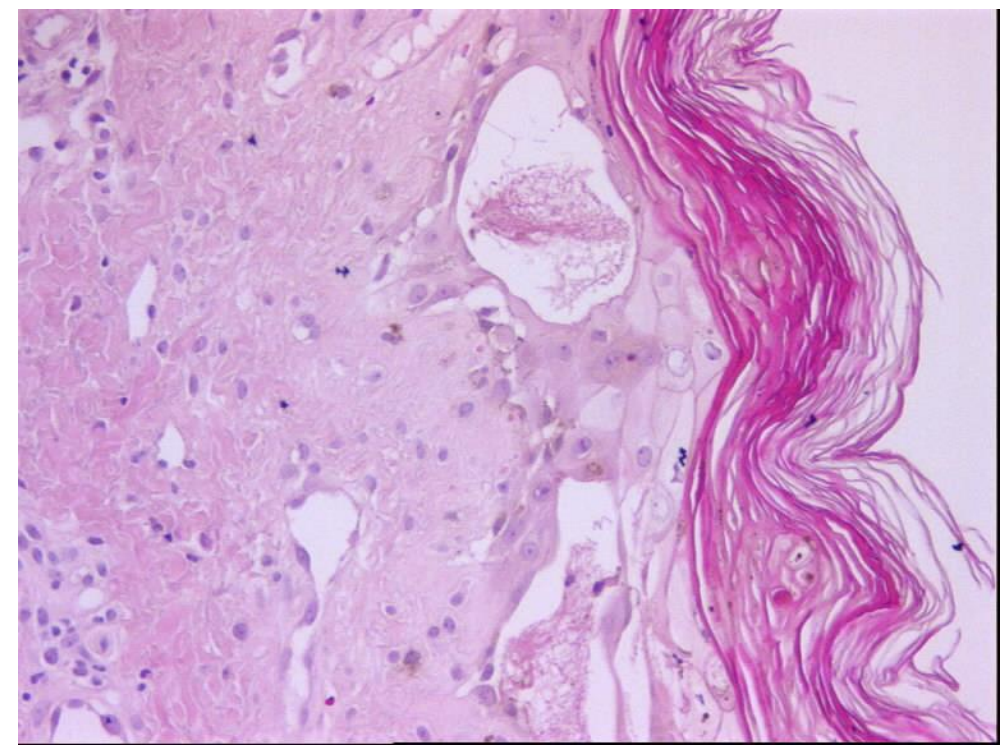

Figure 4. Subepidermal and intraepidermal cell-poor blister formations. (H\&E, x200)

Direct immun florecanse microscopy was negative. At the end of the first week the patient had extensive skin sloughing of multiple body surfaces and mucous membranes as well as fragile skin throughout the remainder of his body. The patient was also tachycardic and hypotensive. Him SCORTEN (TEN scoring system) point was 5. Supportive care measures including hydration, wound debridement, systemic and topical anti- biotics, ophthalmic irrigation and lubrication, nut- ritional support, and pain management were provided. With further progression of symptoms, the patient was admitted to pediatric intensive care unit and he continued to receive aggresive supportive care. As a result of the loss of large areas of skin, our patient developed septicemia. Blood and catheter culture confirmed Klebsiella pneumoniae. The patient died of multiorgan failure at the end of 16 days. To assess the relationship of medication use to adverse effect, the patient's Naranjo probability algorithm score was calculated and supported a probable druginduced reaction (Naranjo score $=5$ ).

\section{Discussion}

TEN accompanied by AGEP has rarely been reported in literature ${ }^{[7]}$. It has been reported that AGEP is usually caused by a reaction to anti- biotics, especially the $\beta$-lactams and macrolides. TEN is frequently caused by antibiotics, nonsteroidal anti-inflammatory drugs (NSAIDs), anticonvulsants ${ }^{[8]}$. Mortality of TEN is high, therefore its differantial diagnosis and appropriate patient care is crucial ${ }^{[3]}$. We present a severe case of TEN, caused by amoxicillin, clinically starting with AGEP like features and marked systemic symptoms with haemodynamic and respiratory instability and resulted by death. In the present case, an acute onset of widespread erythema, pustules and purulent bulla, a large area of epidermal necrolysis, erosion of the mucous membranes and positive Nikolsky's sign over the whole body were present following drug administration. The clinical features appeared similar to AGEP and TEN to a certain extent, but the widespread purulent bulla, which were $>5$ $\mathrm{mm}$ in diameter, emerged on the disease onset and epidermal necrolysis throughout him body meant that the diagnosis differed from that of AGEP. There has been only one case reported drug eruption displaying purulent bulla and epidermal necrolysis. Liu LF at al. showed that the patient suffered a drug eruption following the administration of tetanus antitoxin and sulbenicillin ${ }^{[9]}$. Their patient developed diffuse bright red areas, non-follicular pustules and a generalized 
purulent bulla all over the body, with epidermal necrolysis and the detachment of $46 \%$ of the body surface area [9]. This was first report of drug-related purulent TEN in literature. Our study is the second report about TEN which started by multiple pustules like AGEP in literature. We justify that this case propound a new clinical pattern and have therefore termed it purulent bullous TEN.

We presented this case in 24th EADV Congress, Copenhagen, 2015.

We have conflict with second author Dr Ali Haydar Eskiocak. He gave up to be an author due to enforce of our proffessors. I have written consent form from him about this topic.

\section{Acknowledgments}

We sincerely thanks to Prof Dr Erkan Alpsoy, Dr Ali Haydar Eskiocak and Prof Dr Alphan Küpesiz for their consultation and thanks to Dr. Betül Ünal for her histopathologic examination.

\section{References}

[1] Bouvresse S, Valeyrie-Allanore L, Ortonne N, Konstantinou MP, Kardaun SH, et al. Toxic epidermal necrolysis, DRESS, AGEP: Do overlap cases exist? Orphanet J Rare Dis 2012;7:72.

[2] Kostopoulos TC, Krishna SM, Brinster NK, Ortega-Loayza AG. Acute generalized exanthematous pustulosis: atypical presentations and outcomes. J Eur Acad Dermatol Venereol 2015;29(2):209-14. doi: 10.1111/jdv.12721.
[3] van Hattem S, Beerthuizen GI, Kardaun SH. Severe flucloxacillin-induced acute generalized exanthematous pustulosis (AGEP), with toxic epidermal necrolysis (TEN)-like features: does overlap between AGEP and TEN exist? Clinical report and review of the literature. $\mathrm{Br} \mathrm{J}$ Dermatol 2014;171:1539-45.

[4] Fernando SL. Acute generalised exanthematous pustulosis. Australas J Dermatol 2012;53:87-92.

[5] Peermohamed S, Haber RM. Acute generalized exanthematous pustulosis simulating toxic epidermal necrolysis: a case report and review of the literature. Arch Dermatol 2011;147:697-701.

[6] Lin YF1, Yang CH, Sindy H, Lin JY, Rosaline Hui $C Y$, Tsai $Y C$, et al. Severe cutaneous adverse reactions related to systemic antibiotics. Clin Infect Dis 2014;58(10):1377-85.

[7] Goh TK, Pang SM, Thirumoorthy T, Goh SG. Acute generalised exanthematous pustulosis and toxic epidermal necrolysis induced by carbamazepine. Singapore Med J 2008;49:507-10.

[8] Roujeau JC, Bioulac-Sage P, Bourseau C, et al. Acute generalized exanthematous pustulosis. Analysis of 63 cases. Arch Dermatol 1991;127: 1333-8.

[9] Liu LF, Yan JL, Wang JY, Chen JS, Cai SQ, Lao LM, Zheng M. Purulent bullous epidermal necrolysis: A potential new clinical pattern of drug eruption. Exp Ther Med. 2013;5:10401042.

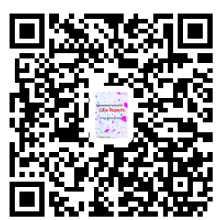

